\title{
A connection selection method for vertical handoff in hybrid wireless environment
}

\begin{abstract}
The next generation wireless networks will consist of heterogeneous access networks from 3G cellular, WiFi, WiMAX to other emerging access technologies. To support the mobile host in the hybrid overlay wireless, a cross-layer vertical handoff control method has been proposed. To efficiently select the optimum network connection, attributes from the multilayer platform is provided to handoff decision component. The proposed algorithm is based on hybrid neuro-fuzzy concept with low packet loss and latency. We have analyzed our implemented model under different scenarios. Results from the experiments indicate the advantages of the proposed scheme.
\end{abstract}

Keyword: Handoff; Mobility management; Wireless network 